\title{
Confort térmico en viviendas de Medellín*
}

\author{
Engelberth Soto-Estrada* \\ Fernanda Álvarez-Carrascal ${ }^{* * *}$ \\ Jairo Gómez-Lizarazo *** \\ Daniel Valencia-Montoya $a^{* * * *}$
}

\author{
Recibido: 01/07/2018 - Aceptado: 21/03/2019 \\ https://doi.org/10.22395/rium.v18n35a4
}

\begin{abstract}
Resumen
El ambiente térmico en una edificación influye en la salud, el bienestar y la productividad de las personas, así como en el consumo energético. La importancia del confort térmico en las edificaciones se reconoce desde hace décadas, no obstante, este aspecto es aún incipiente en la industria de la construcción colombiana, sobre todo en proyectos de vivienda. En esta investigación se analizó el desempeño térmico de tres tipos de vivienda comunes en la ciudad de Medellín. Para ello se calculó la temperatura operativa, el voto medio estimado (PMV) y el porcentaje estimado de insatisfechos (PPD), de acuerdo con la norma ISO 7730:2005. Los resultados mostraron que la inercia térmica de dos de los tres tipos de vivienda examinados no es suficiente para ofrecer un confort térmico adecuado, a pesar del clima templado del Valle de Aburrá. Estos resultados alertan sobre la vulnerabilidad de la población urbana en Colombia ante fluctuaciones extremas de temperatura.
\end{abstract}

Palabras clave: confort térmico; temperatura operativa; temperatura radiante; PMV; PPD.

\footnotetext{
Investigación terminada. Proyecto: Consumo energético doméstico debido a condiciones climáticas. Tiempo de ejecución: 21 meses entre el 2016 y el 2018, financiado por Colciencias (convocatoria 753) y la Universidad EIA.

** Doctor en Administración de Recursos Naturales. Miembro del Grupo de Investigación SITE, programa de Ingeniería Civil, Universidad EIA, Envigado, Colombia. Correo electrónico: engelberth.soto@eia.edu.co. Orcid: https://orcid.org/0000-0002-9572-7063

*** Ingeniera civil. Miembro del Grupo de Investigación SITE, programa de Ingeniería Civil, Universidad EIA, Envigado, Colombia. Correo electrónico: mariafernanda.alvarez@eia.edu.co. Orcid: https://orcid. org/0000-0002-2256-3686

**** Ingeniero industrial. Miembro del Grupo de Investigación GPC, programa de Ingeniería Industrial, Universidad EIA, Envigado, Colombia. Correo electrónico: jairo.gomez@eia.edu.co. Orcid: https://orcid. org/0000-0002-2575-877X

****** Ingeniero ambiental. Miembro del Grupo de Investigación SITE, programa de Ingeniería Industrial, Universidad EIA, Envigado, Colombia. Correo electrónico: daniel.valencia@eia.edu.co. Orcid: https://orcid. org/0000-0003-4842-9693
} 


\title{
Thermal Comfort in Residential Housing in Medellin
}

\begin{abstract}
The indoor thermal environment of buildings influences people's health, well-being and productivity, as well as energy consumption. The importance of thermal comfort in buildings has been recognized for decades. However, this aspect is still incipient in the Colombian construction industry, especially in housing projects. In this research, the thermal performance of three common housing types in the city of Medellin was analyzed. To do this, the operative temperature, the predicted mean vote (PMV) and the predicted percentage of dissatisfied (PPD) were calculated in accordance with ISO 7730:2005. The results showed that the thermal inertia of two of the three types of housing examined was insufficient to offer adequate thermal conditions, despite the temperate climate of the Aburra Valley. These results warn about the vulnerability of urban population in Colombia to extreme temperature fluctuations.
\end{abstract}

Keywords: thermal comfort; operative temperature; radiant temperature; PMV; PPD.

\section{Conforto térmico de habitações em Medellín}

\section{Resumo}

$\mathrm{O}$ ambiente térmico em uma construção influencia na saúde, no bem-estar e na produtividade das pessoas, bem como no consumo energético. A importância do conforto térmico nas edificações é reconhecida há décadas, não obstante, esse aspecto ainda é incipiente na indústria da construção colombiana, sobretudo em projetos de habitação. Nesta pesquisa, o desempenho térmico de três tipos de habitação comuns na cidade de Medellín foi analisado. Para isso, calculou-se a temperatura operativa, o voto médio estimado (PMV) e a porcentagem estimada de insatisfeitos (PPD), de acordo com a norma ISO 7730:2005. Os resultados mostraram que a inércia térmica de dois dos três tipos de habitação examinados não é suficiente para oferecer um conforto térmico adequado, apesar do clima ameno do Valle de Aburrá. Esses resultados alertam sobre a vulnerabilidade da população urbana na Colômbia ante oscilações extremas de temperatura.

Palavras-chave: conforto térmico; temperatura operativa; temperatura radiante; PMV; PPD. 


\section{INTRODUCCIÓN}

La Sociedad de Ingenieros de Calefacción, Refrigeración y Aire Acondicionado de los Estados Unidos de América (Ashrae, por sus siglas en inglés) define al confort térmico como la condición de la mente en la que se expresa satisfacción con el ambiente térmico mediante una valoración subjetiva [1]. Diversos autores [1-8] han señalado que el confort térmico es el resultado de la interacción entre diversas variables físicas, fisiológicas y sicológicas. El diseño arquitectónico de los espacios, la vestimenta, el nivel de actividad física, el clima, la velocidad interna del aire y la percepción sicológica de la temperatura son algunas de las variables investigadas como parte del análisis de ambientes térmicos.

El estudio formal del confort térmico comenzó con el trabajo pionero de A. P. Gagge a principios del siglo XX [9]. En 1936, Gagge publicó el modelo de dos nodos para explicar el balance térmico del cuerpo humano [10], el cual fue la base para estudiar los intercambios de energía entre el cuerpo y su entorno. Posteriormente, P. O. Fanger [3] examinó la relación entre los parámetros físicos del entorno, los parámetros fisiológicos de las personas y su percepción del bienestar térmico; introdujo una escala de siete niveles para valorar el confort térmico, la cual se muestra en la tabla 1.

Tabla 1. Escala de sensación térmica de siete niveles

\begin{tabular}{cc}
\hline Valor & Sensación \\
\hline 3 & Muy caluroso \\
\hline 2 & Caluroso \\
\hline 1 & Ligeramente caluroso \\
\hline 0 & Neutro \\
\hline-1 & Ligeramente fresco \\
\hline-2 & Fresco \\
\hline-3 & Frío \\
\hline
\end{tabular}

Fuente: Aenor [6], Ashrae [1]

Mediante los votos de sujetos de prueba, Fanger elaboró una ecuación que relaciona los parámetros físicos y fisiológicos. Con esta ecuación es posible calcular el "voto medio estimado" (PMV, por sus siglas en inglés), el cual es un índice que predice la sensación térmica de un grupo de personas en un mismo ambiente [1]. El PMV se calcula a través de mediciones de los parámetros físicos del entorno (temperatura del aire, temperatura radiante, velocidad del aire y humedad relativa) y de la tasa metabólica y vestimenta de los habitantes. 
La sensación térmica varía de persona a persona en un mismo espacio y momento, por lo que se considera que incluso cuando el valor del PMV es 0 (neutro), existe un porcentaje de personas insatisfechas. Debido a ello, Fanger también propuso un índice para la evaluación de la incomodidad o insatisfacción térmica, denominado "porcentaje estimado de insatisfechos" (PPD, por sus siglas en inglés). El PPD indica el porcentaje de personas que, probablemente, sentirán demasiado calor o demasiado frío en un ambiente determinado [6]. El PPD es una función del PMV, como se indica en la figura 1.

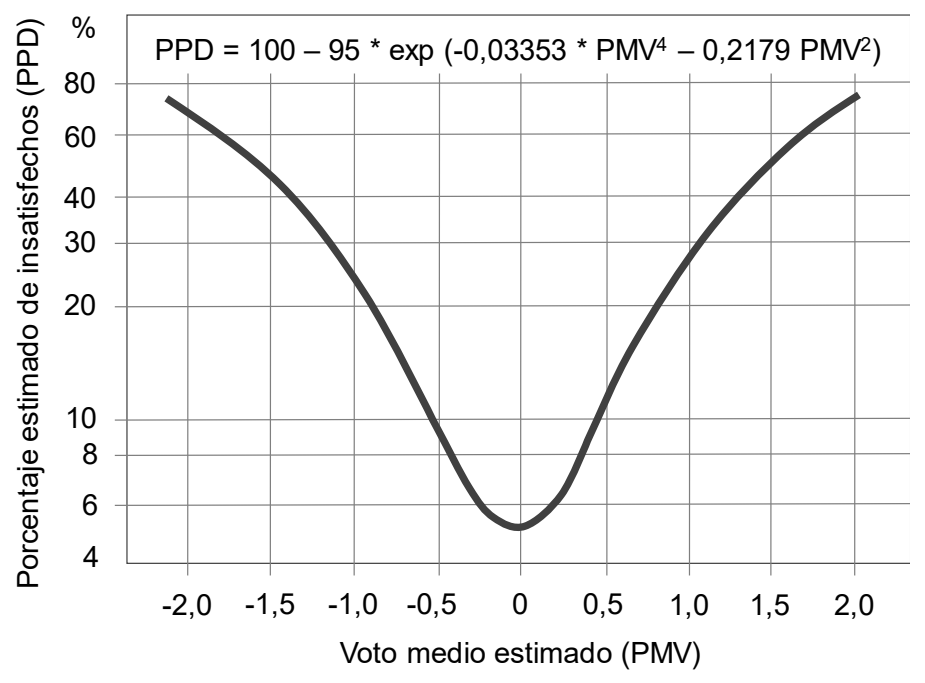

Figura 1. PPD en función del PMV

Fuente: Ashrae [1]

La temperatura operativa óptima de un espacio (equivalente a PMV $=0$ ) depende primordialmente de la actividad física de sus ocupantes y de la ropa que lleven puesta [6]. La norma ISO 7730:2005 [6] clasifica los ambientes térmicos en tres categorías: A, B y C; para cada una establece temperaturas óptimas e intervalos de temperatura aceptables. Para espacios en los cuales se desarrollan actividades sedentarias, como las realizadas en una vivienda, la norma ISO 7730:2005 recomienda temperaturas óptimas de $24 \pm 1,0{ }^{\circ} \mathrm{C}$ para la categoría A, $24 \pm 1,5^{\circ} \mathrm{C}$ para la B, y $24 \pm 2,5^{\circ} \mathrm{C}$ para la C [6]. Por su parte, el Estándar 55-2017 de Ashrae [1] señala que la temperatura operativa aceptable para espacios naturalmente ventilados, en los cuales se desarrollan actividades de reposo o sedentarias, por personas con vestimenta de uso diario normal (ropa interior, camisa, pantalón, calcetines y zapatos), se encuentra entre los límites mostrados en la figura 2. 


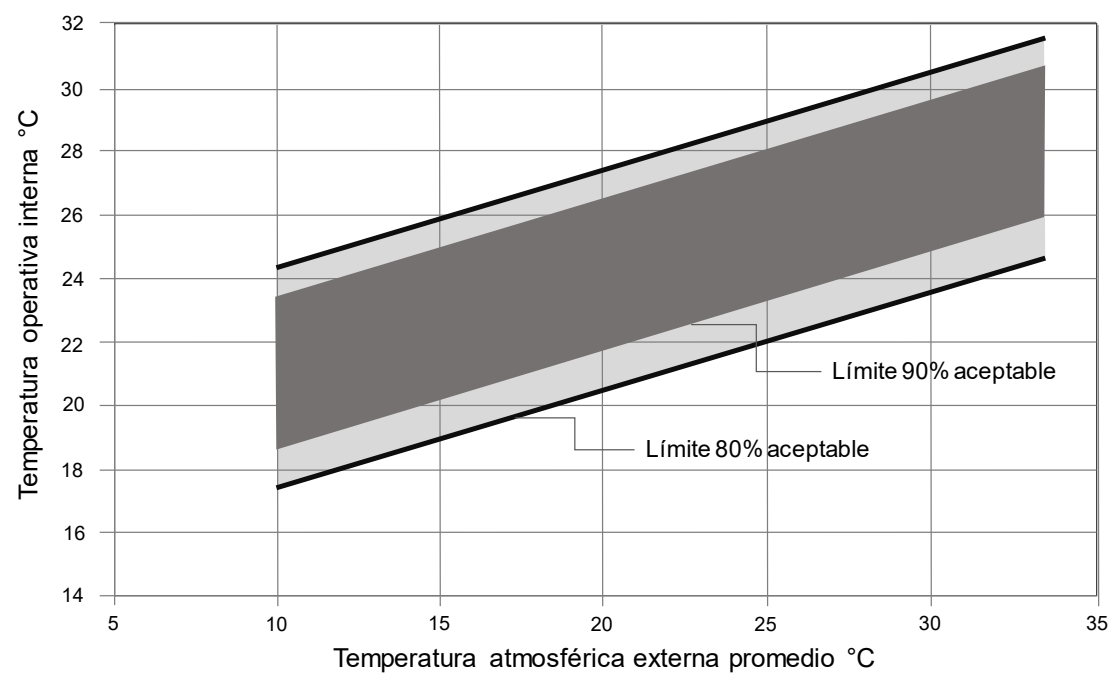

Figura 2. Intervalo de temperatura operativa aceptable para espacios acondicionados naturalmente Fuente: Ashrae [1]

En Colombia, la mayor parte de los proyectos de vivienda no consideran aspectos de confort térmico durante su diseño. De acuerdo con el Consejo Colombiano de Construcción Sostenible [11], en el país la mayoría de los proyectos "verdes" son de uso comercial o de oficinas, registrándose 350 proyectos con certificado LEED (Leadership in Energy and Environmental Design) hasta diciembre del 2017. Según la misma fuente, las empresas del sector consideran que la inclusión de aspectos "sostenibles" genera mayores costos, sobre todo en proyectos de vivienda.

En este artículo se presenta un análisis del desempeño térmico de tres tipos de vivienda comunes en el Valle de Aburrá. Cada vivienda se instrumentó con sensores de temperatura atmosférica, temperatura radiante, velocidad del viento y humedad para calcular su temperatura operativa y, con ello, el confort térmico que ofrecen a sus habitantes. El documento se estructura de la siguiente manera: en la sección que sigue se explican los materiales y métodos utilizados, posteriormente se presentan y discuten los resultados $\mathrm{y}$, finalmente, se resumen las conclusiones del análisis.

\section{METODOLOGÍA}

El PMV se calculó con el procedimiento indicado en la norma ISO 7730:2005, ecuaciones 1-4 [6]. Los casos de estudio se instrumentaron con los sensores de la tabla 2 para medir los parámetros físicos: temperatura del aire (interna y externa), temperatura radiante, velocidad del aire y humedad relativa (interna y externa). Para 
la selección de los sensores se tomó en cuenta lo indicado en la norma ISO 7726:1998 [12]. Las mediciones se realizaron con una frecuencia de cinco minutos a lo largo de catorce días consecutivos en cada caso de estudio. Adicionalmente, durante ese periodo se encuestó a los habitantes de las viviendas para obtener información sobre su nivel de actividad física, vestimenta, sensación térmica, edad y género, registrándose la hora y fecha de cada respuesta. Las respuestas se diligenciaron en un formato físico que se dejó a disposición de los ocupantes de las viviendas; adicionalmente, se habilitó una encuesta en una plataforma en línea para los habitantes que preferían esa opción.

Tabla 2. Instrumentos utilizados para medir parámetros físicos

\begin{tabular}{lll}
\hline \multicolumn{1}{c}{ Sensor } & Variable medida & Frecuencia de medición \\
\hline KIMO KH50 & $\begin{array}{l}\text { Temperatura seca }\left({ }^{\circ} \mathrm{C}\right) \\
\text { Humedad relativa }(\%)\end{array}$ & \\
\cline { 1 - 2 } $\begin{array}{l}\text { KIMO KT 220N con sonda termopar K } \\
\text { con globo negro BN 150-45 }\end{array}$ & Temperatura de globo $\left({ }^{\circ} \mathrm{C}\right)$ & $\begin{array}{l}\text { Cada } 5 \text { minutos durante 14 días } \\
\text { para cada caso de estudio }\end{array}$ \\
\cline { 1 - 2 } Anemómetro tipo molino CEM DT-187 & Velocidad del aire $(\mathrm{m} / \mathrm{s})$ & \\
\hline
\end{tabular}

Fuente: elaboración propia

$$
\begin{aligned}
& P M V= {[0,303 * \exp (-0,036 * M)+0,028] * } \\
&\left\{\begin{array}{l}
(M-V)-3,05 * 10^{-3} *\left[5733-6,99 *(M-V)-P_{a}\right]-0,42 \\
*[(M-V)-58,15]-1,7 * 10^{-5} * M\left(5867-P_{a}\right)-0,0014 * M \\
*\left(34-t_{a}\right)-3,96 * 10^{-8} * f_{c l} *\left[\left(t_{c l}+273\right)^{4}-\left(\overline{t_{r}}+273\right)^{4}\right]-f_{c l} \\
* h_{c} *\left(t_{c l}-t_{a}\right)
\end{array}\right. \\
& t_{c l}=35,7-0,028 *(M-W)-I_{c l} * \\
&\left\{\begin{array}{r}
3,96 * 10^{-8} * f_{c l} *\left[\left(t_{c l}+273\right)^{4}-\left(\overline{t_{r}}+273\right)^{4}\right]+f_{c l} * h_{c} *\left(t_{c l}-t_{a}\right.
\end{array}\right\} \\
& h_{c}=2,38\left|t_{c l}-t_{a}\right|^{0,25} \quad \text { para } \quad 2,38 *\left|t_{c l}-t_{a}\right|^{0,25}>12,1 * \sqrt{v_{a r}} \\
& h_{c}=12,1 * \sqrt{v_{a r}} \quad \text { para } \quad 2,38 *\left|t_{c l}-t_{a}\right|^{0,25}<12,1 * \sqrt{v_{a r}} \\
& f_{c l}=1,00+0,290 I_{c l} \quad \text { para } \quad I_{c l} \leq 0,078 m^{2} * K / W \\
& f_{c l}=1,05+0,645 I_{c l} \quad \text { para } \quad I_{c l}>0,078 m^{2} * K / W
\end{aligned}
$$


Donde:

$M$ : tasa metabólica $\left(\mathrm{W} / \mathrm{m}^{2}\right)$, estimada con los datos de las encuestas y la tabla B.1 de la norma ISO 7730:2005

$W$ : potencia mecánica efectiva $\left(\mathrm{W} / \mathrm{m}^{2}\right)$, considerada 0 según la norma ISO 7730:2005

$I_{c l}$ : aislamiento de la ropa $\left(\mathrm{m}^{2 *} \mathrm{~K} / \mathrm{W}\right)$, estimado con los datos de las encuestas y la tabla C.1 de la norma ISO 7730:2005

$f_{c l}$ : factor de superficie de la ropa, 1,15 correspondiente a vestimenta completa liviana [13]

$t_{a}$ : temperatura del aire $\left({ }^{\circ} \mathrm{C}\right)$, medida en campo cada 5 minutos durante 14 días para cada caso de estudio

$\overline{t_{r}}$ : temperatura radiante media $\left({ }^{\circ} \mathrm{C}\right)$, medida en campo cada 5 minutos durante 14 días para cada caso de estudio

$v_{a r}$ : velocidad relativa del aire $(\mathrm{m} / \mathrm{s})$, se consideró como $0,1 \mathrm{~m} / \mathrm{s}$, tabla A.5 de la norma ISO 7730:2005, debido al fallo de los anemómetros instalados

$p_{a}$ : $\quad$ presión parcial de vapor de agua $(\mathrm{Pa})$, medida en campo cada 5 minutos durante 14 días para cada caso de estudio

$h_{c}$ : coeficiente de transmisión de calor por convección $\left[\mathrm{W} /\left(\mathrm{m}^{2 *} \mathrm{~K}\right)\right]$

$t_{c l}: \quad$ temperatura de la superficie de la ropa $\left({ }^{\circ} \mathrm{C}\right)$

El PDD se calculó mediante la ecuación 5 [6]:

$$
P P D=100-95 * \exp \left(-0,03353 * P M V^{4}-0,2179 * P M V^{2}\right)
$$

Donde:

PPD: porcentaje estimado de insatisfechos

$P M V:$ voto medio estimado

En cada caso de estudio se instalaron sensores de temperatura seca y humedad relativa en la sala, una habitación, cocina y en el exterior de la vivienda. El sensor externo se protegió con un escudo de radiación. El termómetro de globo negro se instaló en la sala. 
La temperatura radiante media se calculó con la ecuación 6 [13]:

$$
\overline{t_{r}}=\left[\left(t_{g}+273\right)^{4}+\frac{1,10^{*} 10^{8} v_{a}^{0,6}}{\varepsilon D^{0,4}}\left(t_{g}-t_{a}\right)\right]^{1 / 4}-273
$$

Donde:

$\overline{t_{r}}:$ temperatura radiante media $\left({ }^{\circ} \mathrm{C}\right)$

$t_{g:}$ temperatura de globo $\left({ }^{\circ} \mathrm{C}\right)$

$v_{a}$ : velocidad del aire $(\mathrm{m} / \mathrm{s})$

$t_{a}$ : temperatura del aire $\left({ }^{\circ} \mathrm{C}\right)$

$D:$ diámetro del globo $(\mathrm{m})$

$\varepsilon$ : emisividad, 0,95 para globos negros [13]

La temperatura operativa de las viviendas se calculó utilizando la ecuación 7 [1]:

$$
t_{o}=A t_{a}+(1-A) \overline{t_{r}}
$$

Donde:

$t_{o:}$ temperatura operativa $\left({ }^{\circ} \mathrm{C}\right)$

$t_{a}$ : temperatura media del aire $\left({ }^{\circ} \mathrm{C}\right)$

$\overline{t_{r}}$ : temperatura radiante media $\left({ }^{\circ} \mathrm{C}\right)$

A: 0,5 para espacios con velocidad del aire menor a $0,2 \mathrm{~m} / \mathrm{s},[1]$

Con los datos recopilados se calculó la temperatura operativa, el PMV y el PPD cada 5 minutos, en tanto que la percepción de confort de los habitantes (PMV) se graficó en la jornada (mañana, tarde o noche) y día en que fue emitida. En general, los ocupantes de las viviendas respondieron el cuestionario de forma esporádica, como se aprecia en la figura 12. Cabe aclarar que los anemómetros fallaron en el almacenamiento de la información, por lo que se tuvo que suponer un valor típico de velocidad de viento, como se indica en la norma ISO 7730:2005.

\section{RESULTADOS Y DISCUSIÓN}

Se analizaron diez viviendas de distintos estratos socioeconómicos y características constructivas. En este artículo se discuten los resultados de tres de ellas, por ser representativas de las edificaciones de la región metropolitana del Valle de Aburrá. 
En el texto se mencionaron con el número consecutivo asignado en el orden en el que fueron analizadas.

Las figuras 3 y 4 muestran la temperatura operativa del caso 7 a lo largo de los 14 días de análisis, del 14 al 28 de mayo del 2018, días en los cuales se presentaron condiciones cambiantes de sol y lluvia. La figura 3 presenta la temperatura operativa durante las 24 horas del día para cada uno de los 14 días de medición y la temperatura atmosférica externa promedio. En la figura 3 también se indica, con color gris, la zona de confort para el ambiente térmico C de acuerdo con la norma ISO 7730:2005. La figura 4 muestra los datos de temperatura operativa y temperatura atmosférica externa de forma consecutiva durante todo el periodo de análisis.

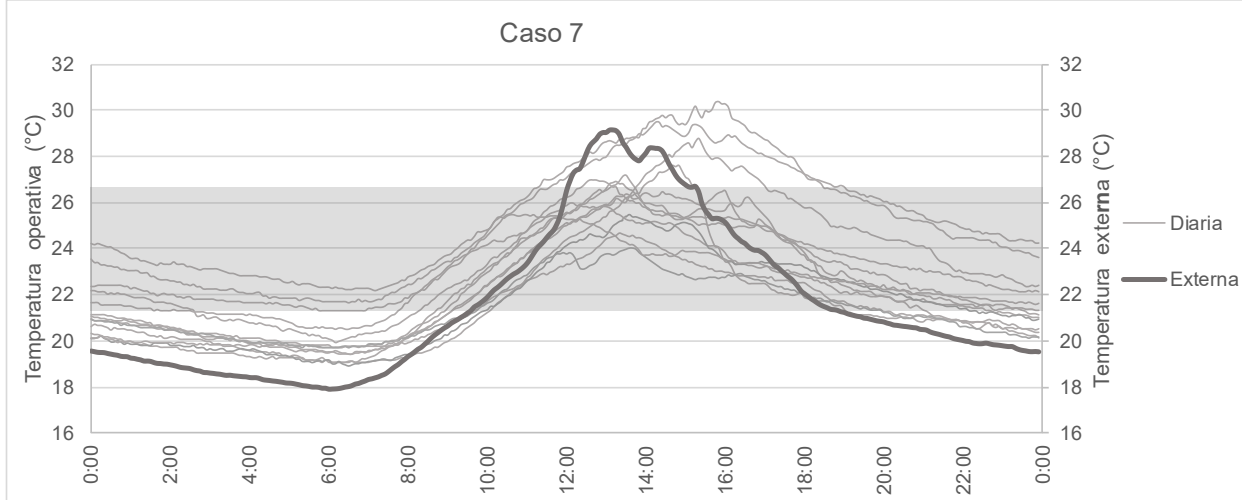

Figura 3. Temperatura operativa diaria del caso de estudio 7 y temperatura externa promedio Fuente: elaboración propia

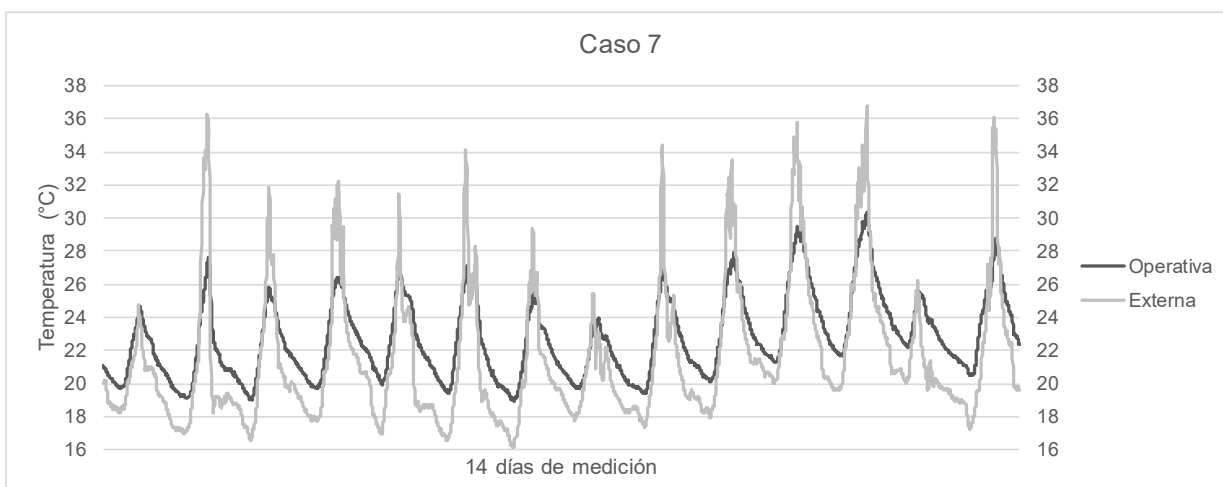

Figura 4. Temperaturas promedio, operativa y externa, del caso de estudio 7 a lo largo de los 14 días de análisis

Fuente: elaboración propia 
El caso de estudio 7 se localiza en la parte baja del Valle de Aburrá. Tiene un área construida de $85,2 \mathrm{~m}^{2}$, paredes de mampostería en arcilla recubiertas con mortero, estuco y pintura, pisos de baldosa cerámica y techo de machimbre recubierto con tela asfáltica y tejas de arcilla. Se puede afirmar que es un apartamento típico de último piso, en este caso el sexto, de un edificio pequeño en el Valle de Aburrá (ver figura 5).

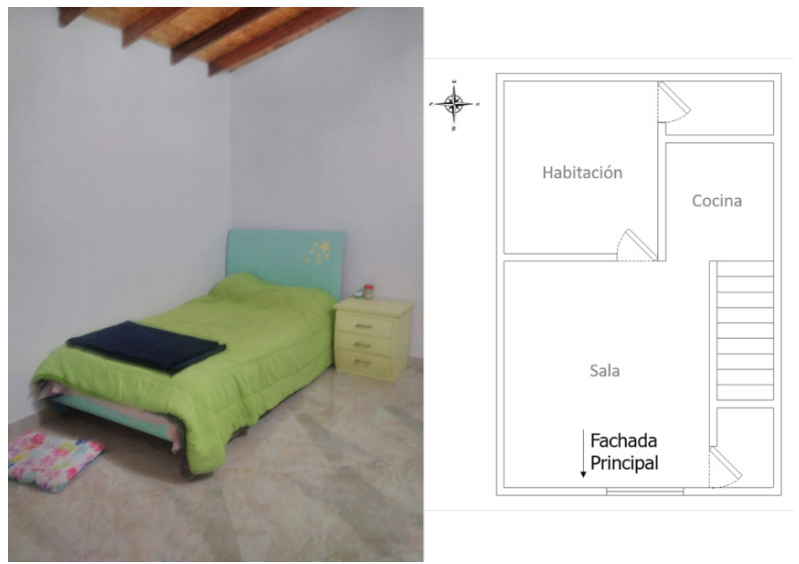

Figura 5. Vista interna y distribución del caso de estudio 7

Fuente: elaboración propia

La temperatura operativa del caso 7 se situó entre $18,9{ }^{\circ} \mathrm{C}$ y $30,4{ }^{\circ} \mathrm{C}$ durante el periodo de análisis. La temperatura externa promedio fluctuó entre $17,9{ }^{\circ} \mathrm{C}$ y $29,2{ }^{\circ} \mathrm{C}$, como se aprecia en la figura 3 (línea oscura). No obstante, la temperatura externa mínima registrada durante los 14 días de medición fue de $16,2{ }^{\circ} \mathrm{C}$ y la máxima de $36,2{ }^{\circ} \mathrm{C}$, como se muestra en la figura 4 . De acuerdo con los valores de temperatura operativa calculados, esta vivienda cumplió el 48,4\% del tiempo con lo indicado por la norma ISO 7730:2005 para el ambiente térmico C (zona gris en la figura 3), el más laxo: $24 \pm 2,5^{\circ} \mathrm{C}$, observándose mayores necesidades de calefacción que de refrigeración durante los días analizados.

La inercia térmica del caso 7, entendida como la capacidad de los elementos opacos (muros, techos y pisos) para amortiguar y distribuir en el tiempo las fluctuaciones de temperatura [15], produjo que la temperatura operativa mínima promedio fuera $2,1{ }^{\circ} \mathrm{C}$ más caliente que la mínima externa, en tanto que la operativa máxima promedio fue de $4,9{ }^{\circ} \mathrm{C}$ más fría que la exterior. Durante los 14 días de medición, la amortiguación máxima de temperaturas mínimas fue de $3,2^{\circ} \mathrm{C}$ y de $-8,7{ }^{\circ} \mathrm{C}$ para temperaturas máximas, como se aprecia en la figura 4 . En lo que corresponde al desfase temporal entre la temperatura operativa y la temperatura externa, es decir, lo que tardan en manifestarse los cambios externos de temperatura al interior de la vivienda, en la figura 4 se puede observar que las temperaturas mínimas y máximas, tanto operativas como 
externas, ocurrieron prácticamente al mismo tiempo, por lo que se puede afirmar que el desempeño térmico del caso 7 fue limitado.

Ulgen [16] señala que, dependiendo de las propiedades termofísicas y del espesor de los elementos opacos que componen una edificación, se pueden observar desfases temporales de hasta 12 horas entre las temperaturas externas e internas. Tuohy et al. [17] mencionan que una construcción con buen aislamiento térmico ( $U=0,1$ $\mathrm{W} / \mathrm{m}^{2} \mathrm{~K}$ ) y sistema de recuperación de calor puede "sobrevivir" una semana sometida a temperaturas externas de $0{ }^{\circ} \mathrm{C}$ sin necesidad de calefacción adicional.

En la literatura científica no existe un consenso sobre la inercia térmica ideal para edificaciones. En general, se considera que para la mayoría de las edificaciones y climas, entre mayor sea la masa térmica mejor será el confort y menor el consumo energético [15]. No obstante, algunos investigadores [18-19] señalan que, en climas cálidos, como el de Medellín, la demanda energética para refrigeración podría incrementar al aumentar la inercia térmica. Esto se debe a que los elementos opacos almacenan una mayor cantidad de energía durante el día, la disipan en la noche y con ello incrementan la demanda de refrigeración en las horas en las que hay más ocupantes.

Como se ha indicado anteriormente, en esta investigación se examinaron distintas tipologías constructivas. Al comparar su desempeño térmico, se puede afirmar que la solución constructiva del caso 7 no es la mejor para las condiciones del Valle de Aburrá, pero tampoco la peor, como se mostrará más adelante. Se considera que el techo de machimbre reduce la inercia térmica de las viviendas y, con ello, su desempeño térmico.

Las figuras 8 a 11 muestran la temperatura operativa de los casos de estudio 1 y 6 . El caso 1 corresponde a un apartamento de estrato $6\left(231 \mathrm{~m}^{2}\right)$ construido con muros, pisos y techo de concreto con diferentes recubrimientos y pisos de madera (ver figura 6), en tanto que el caso 6 pertenece una vivienda de estrato $1\left(18 \mathrm{~m}^{2}\right)$ construida con materiales ligeros: muros de madera y techo de láminas de zinc (ver figura 7).

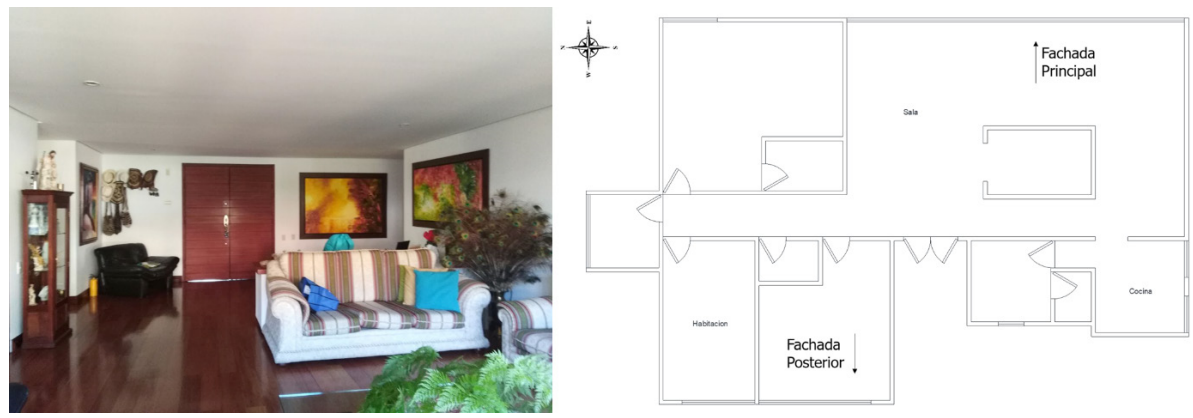

Figura 6. Vista interna y distribución del caso de estudio 1

Fuente: elaboración propia 

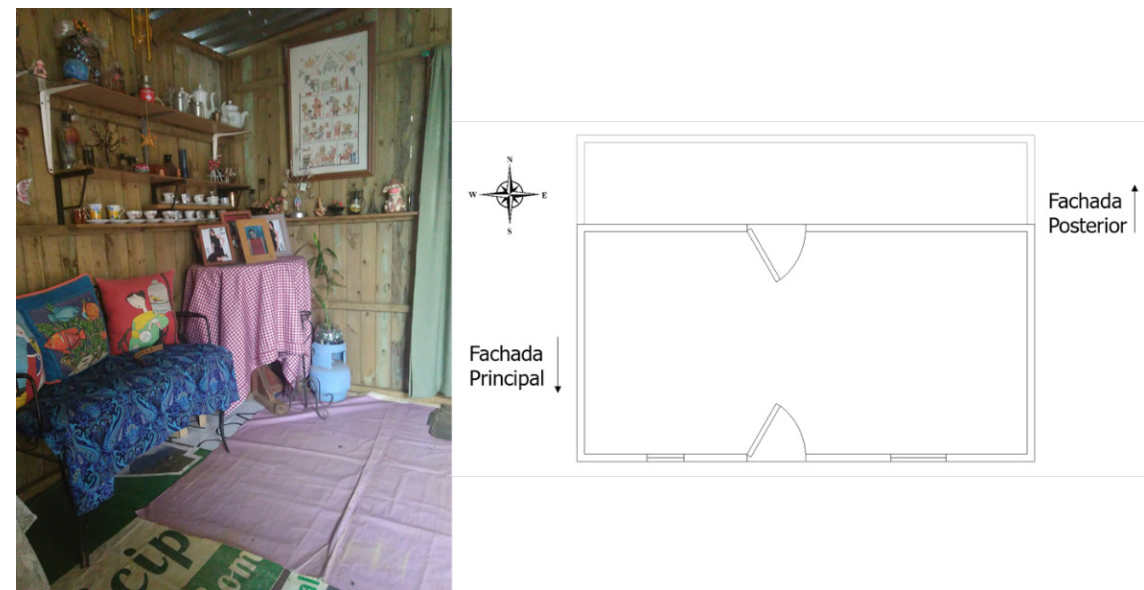

Figura 7. Vista interna y distribución del caso de estudio 6

Fuente: elaboración propia

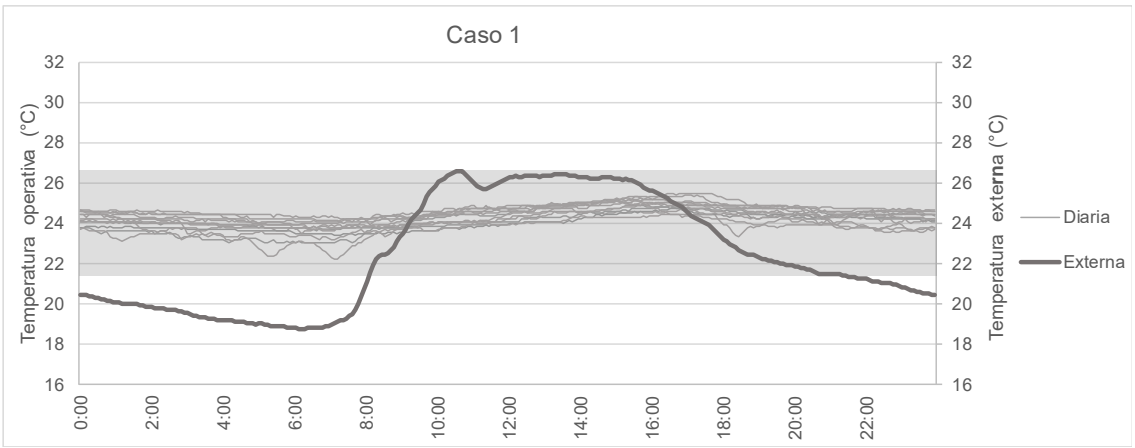

Figura 8. Temperatura operativa diaria del caso de estudio 1 y temperatura externa promedio Fuente: elaboración propia

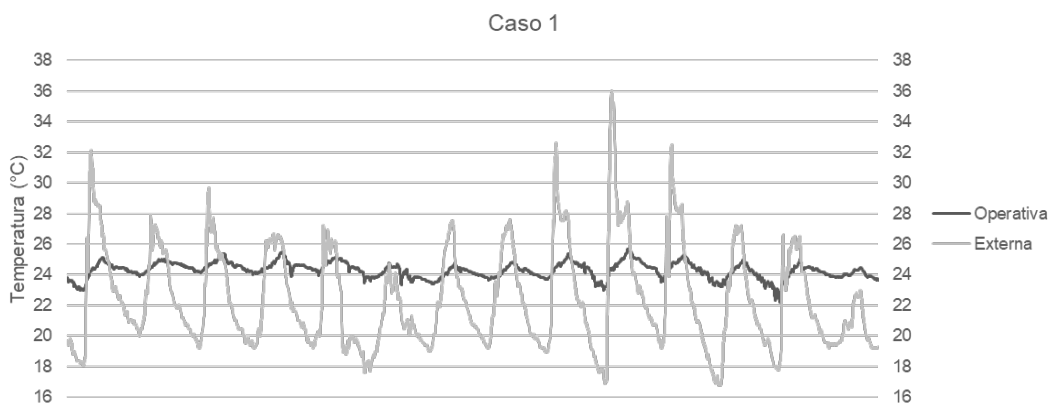

Figura 9. Temperaturas promedio, operativa y externa, del caso de estudio 1 a lo largo de los 14 días de análisis

Fuente: elaboración propia 


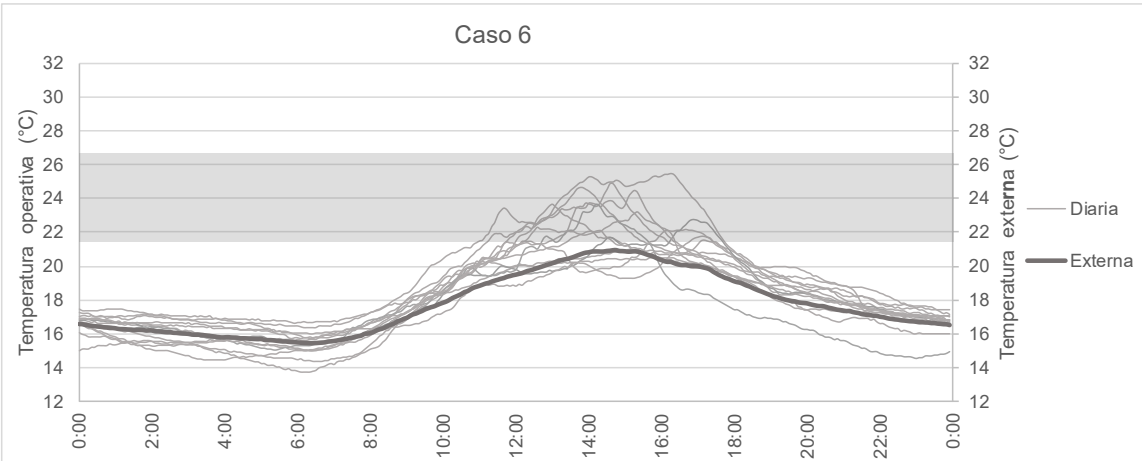

Figura 10. Temperatura operativa diaria del caso de estudio 6 y temperatura externa promedio Fuente: elaboración propia

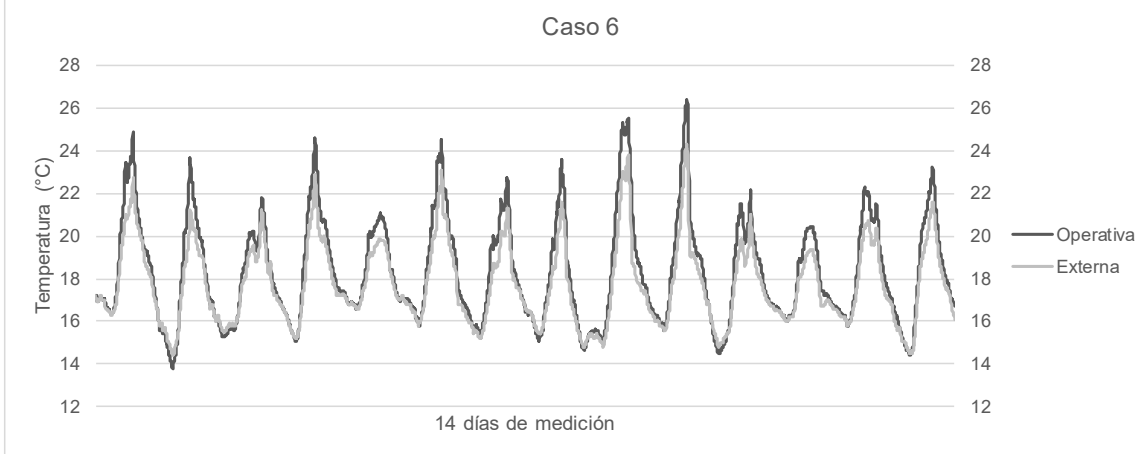

Figura 11. Temperaturas promedio, operativa y externa, del caso de estudio 6 a lo largo de los 14 días de análisis Fuente: elaboración propia

Las figuras anteriores muestran desempeños térmicos opuestos. El caso 1, con mayor inercia térmica por los elementos opacos de concreto, tuvo un mejor desempeño. La temperatura operativa permaneció dentro de los límites que indica la norma ISO 7730:2005 los 14 días de medición, como se aprecia en la figura 8. La temperatura operativa mínima fue, en promedio, $4,9^{\circ} \mathrm{C}$ más caliente que la temperatura mínima exterior, en tanto que la operativa máxima $3,6^{\circ} \mathrm{C}$ más fría que la máxima exterior. La amortiguación máxima fue de $6,4^{\circ} \mathrm{C}$ al comparar temperaturas mínimas (operativa externa) y $-10,3{ }^{\circ} \mathrm{C}$ para las temperaturas máximas a lo largo del periodo de análisis. El caso 1 registró un desfase temporal promedio de 3,7 horas entre los registros de temperaturas máximas exteriores y las máximas operativas, como se observa en la figura 9.

El desempeño del caso de estudio 6 es contrario al anterior (ver figuras 10 y 11). Su inercia térmica no fue suficiente para alcanzar una temperatura operativa adecuada, ni 
para amortiguar las fluctuaciones térmicas. Más aún, la temperatura operativa mínima promedio fue menor que la mínima externa $\left(-0,1^{\circ} \mathrm{C}\right)$, mientras que la temperatura operativa máxima en promedio, fue $1,6^{\circ} \mathrm{C}$ mayor que la temperatura máxima externa. Esta edificación tampoco generó desfases temporales de temperatura. Las temperaturas operativas reaccionaron instantáneamente ante los cambios externos, por lo que se puede afirmar que el funcionamiento térmico de la vivienda depende completamente del clima del Valle de Aburrá. Los días de medición del caso 6 fueron nublados y lluviosos, lo que originó que solo el 8,6\% del tiempo se tuvieran temperaturas operativas dentro del intervalo que señala la norma ISO 7730:2005 para ambientes térmicos de categoría C.

Este resultado muestra la vulnerabilidad de la población que habita en edificaciones como el caso 6, la cual se encuentra a expensas de las condiciones climáticas. Scott [20], así como Campbell-Lendrum y Corvalán [21] han alertado sobre esta problemática. Estos investigadores señalan que la población vulnerable ante fluctuaciones extremas de temperatura crecerá durante las próximas décadas en los países en vías de desarrollo. Lo anterior se debe a la expansión acelerada de las ciudades en estos países, la cual no se muestra acorde con su crecimiento económico, dando como resultado el incremento de cinturones de miseria. Aunado a ello, la ocurrencia de condiciones climáticas adversas podría incrementar debido al calentamiento global, la aparición de olas de calor y el aumento en la intensidad de las islas de calor urbanas.

La figura 12 presenta el PMV calculado para los casos de estudio discutidos en este trabajo, así como la sensación térmica expresada por sus habitantes.

En el caso 7 se obtuvieron valores de PMV entre 1 (ligeramente caluroso) y -1 (ligeramente fresco) la mayor parte del tiempo, lo que coincidió, en términos generales, con la opinión de los habitantes. El PPD promedio fue 18,8 \%, que se puede atribuir a la variación de temperatura operativa durante los días de estudio.

El caso 1 fue el de mejor desempeño. El PMV fue cercano a 0 (neutro) durante los 14 días de medición y el PPD promedio fue de $5 \%$. No obstante, como se aprecia en la figura 12, en el caso de estudio 1 solo el $53 \%$ de las respuestas coincidió con el PMV calculado, aunque ninguna de las respuestas discordantes se puede considerar como una insatisfacción térmica (PMV $=3$ o -3 ), de manera que el PPD calculado fue congruente con la percepción térmica de los habitantes.

Por último, el caso de estudio 6 generó PMV de -1 (ligeramente fresco) la mayor parte del tiempo y un PPD promedio de 22,3\%. En general, los habitantes de esta vivienda expresaron sensaciones térmicas superiores al PMV calculado (tanto hacia arriba como hacia abajo), lo que significa que sienten más calor o frío dentro de la 
vivienda que fuera de ella. Este resultado es otro indicio del bajo desempeño térmico de esta edificación.

Caso 7

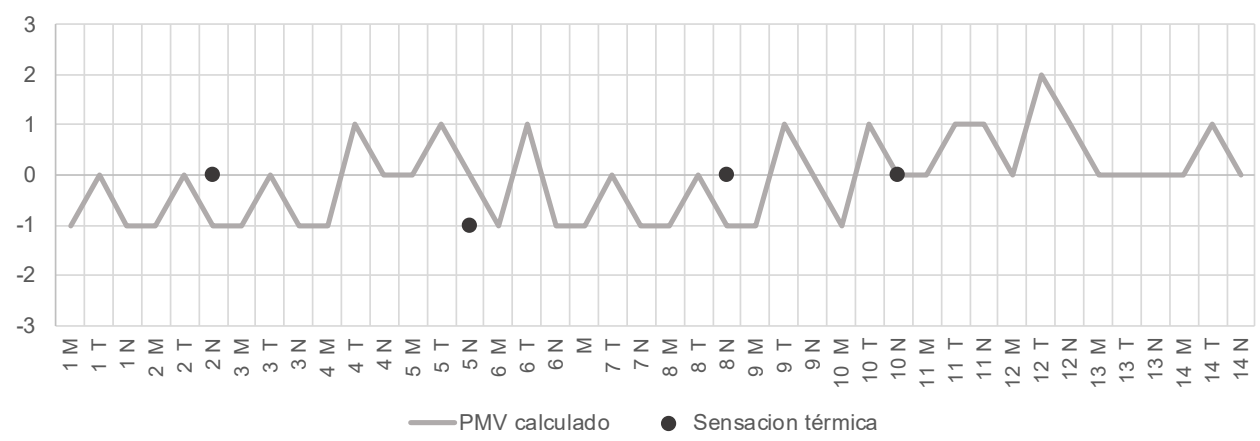

Caso 1

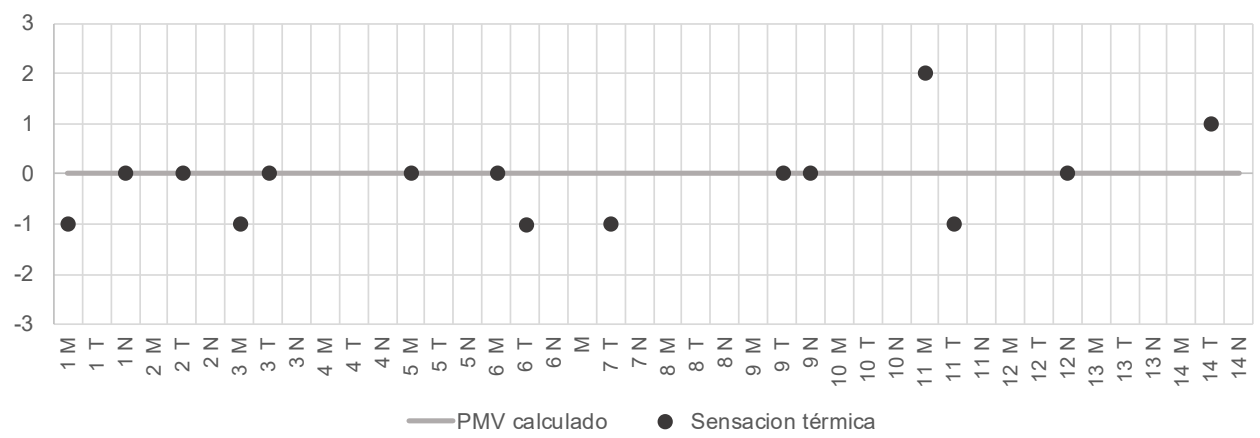

Caso 6

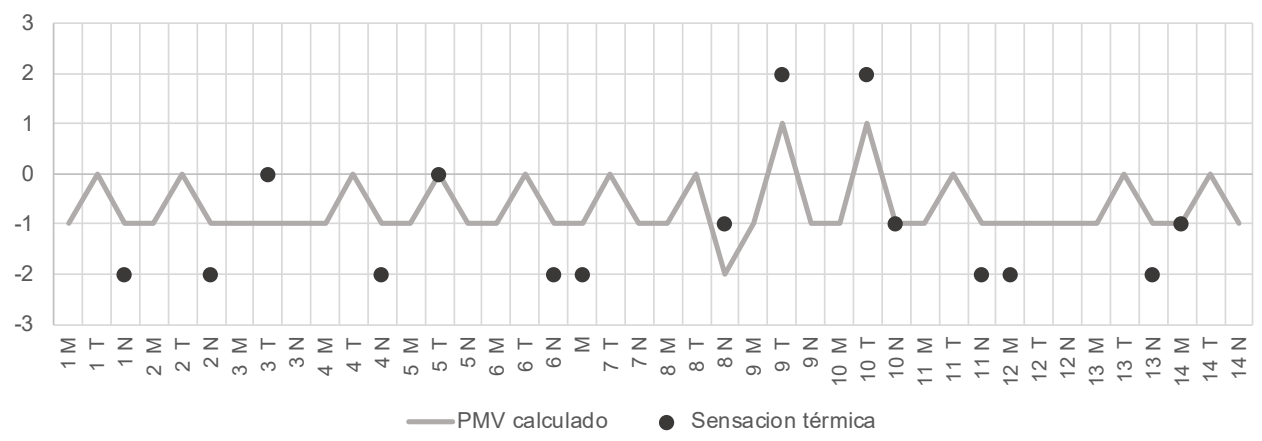

Figura 12. PMV calculado y sensación térmica reportada en los casos 7, 1 y 6

Fuente: elaboración propia 


\section{CONCLUSIONES}

Los resultados resaltan el papel de la inercia térmica en el desempeño de las edificaciones, el cual es notorio incluso en climas templados como el del Valle de Aburrá. Las temperaturas operativas, el PMV y el PPD calculados señalan que la edificación con elementos opacos de concreto es la de mejor desempeño, en tanto que la vivienda construida con materiales ligeros (madera y láminas de zinc) -común en los barrios de estratos 1 y 2 del área metropolitana- ofrece un confort térmico limitado, el cual depende completamente de la temperatura externa. La combinación tradicional de materiales: muros de ladrillo de arcilla y techo de machimbre, también genera un desempeño térmico reducido, lo que se puede deber a la baja inercia térmica de los materiales del techo.

Estos resultados indican que los proyectos de vivienda se deben mejorar para incrementar el confort térmico de la población en el Valle de Aburrá, por ejemplo, aislando térmicamente los techos de machimbre, pero también alertan sobre la vulnerabilidad de la población ante fluctuaciones extremas de temperatura, sobre todo en donde se utilizan materiales ligeros para edificar.

En esta línea de trabajo se continuarán examinando más casos de estudio para corroborar y comparar los desempeños térmicos de distintas combinaciones de materiales constructivos. Adicionalmente, se analizará la influencia de otras variables, tales como la orientación de la vivienda, el tamaño y tipo de ventanas y la presencia de vegetación en el entorno de la edificación. También se trabajará en el desarrollo de plataformas interactivas para recopilar la sensación térmica de los ocupantes de las viviendas. Finalmente, se investigará la influencia del desempeño térmico en el consumo energético de la ciudad.

\section{AGRADECIMIENTOS}

Los autores desean agradecer a la Universidad EIA y al Departamento Administrativo de Ciencia, Tecnología e Innovación de Colombia-Colciencias- por el apoyo otorgado para la realización de este proyecto.

\section{REFERENCIAS}

[1] Thermal Environmental Conditions for Human Occupancy, ANSI/Ashrae Standard 55-2017, American Society of Heating, Refrigerating and Air-Conditioning Engineers, 2017.

[2] H. M. Vernon y C. G. Warner, "The influence of the humidity of the air on capacity for work at high temperatures," Journal of Industrial Hygiene, vol. 32, n. 3, pp. 431-463, 1932. DOI: https://doi.org/10.1017/S0022172400018167 
[3] P. O. Fanger, "Calculations of thermal comfort: introduction of a basic comfort equation," Ashrae Transactions, vol. 73, n. ${ }^{\circ}$ 2, pp. 1-4, 1967.

[4] A. P. Gagge et al., "An effective temperature scale based on a simple model of human physiological regulatory response," Ashrae Transactions, vol. 77, pp. 247-262, 1971.

[5] L. G. Berglund y A. P. Gobelets, "Subjective human response to low-level air current and asymmetric radiation," Ashrae Transactions, vol. 93, pp. 497-523, 1987.

[6] Ergonomía del ambiente térmico, Aenor, Asociación Española de Normalización y Certificación, Estándar UNE-EN ISO 7730, 2006.

[7] H. Frederick y J. Rohles, "Temperature and temperament. A psychologist looks at comfort," Ashrae Journal, vol. 49, pp. 14-22, 2007.

[8] J. Anderson y M. French, "Sustainability as promoting well-being: psychological dimensions of thermal comfort," [En línea], Disponible: https://www.irbnet.de/daten/iconda/CIB20934. pdf, 2010 .

[9] K. Fabbri, Indoor thermal comfort perception. A questionnaire approach focusing on children, Switzerland: Springer International Publishing, 147 p., 2015. DOI: https://doi. org/10.1007/978-3-319-18651-1

[10] A. P. Gagge, "The linearity criterion as applied to partitional calorimetry," American Journal of Physiology, vol. 116, n. ${ }^{\circ}$ 3, pp. 656-668, 1936. DOI: https://doi.org/10.1152/ ajplegacy.1936.116.3.656.

[11] CCCS, "Consejo Colombiano de Construcción Sostenible. La vivienda sostenible se abre espacio en el país,” Revista Portafolio, [En línea], Disponible: https://www.cccs.org.co/wp/ download/la-vivienda-sostenible-abre-espacio-en-el-pais/?wpdmdl=15044, 2017

[12] Eronomics of the termal environment - Instruments for measuring physical quantities, International Organization for Standarization, , Estándar ISO 7726, 1998.

[13] F. Fernández-García, "Fundamentos físicos y métodos de evaluación del confort climático en los estudios de bioclimatología humana," presentado en VI Reunión Nacional de Climatología, Santiago de Compostela, 2000.

[14] Ashrae, 2001 Ashrae Handbook: fundamentals, Atlanta: American Society of Heating, Refrigerating and Air-Conditioning Engineers, 544 p, 2001.

[15] S. Verbeke y A. Audenaert, "Thermal inertia in buildings: A review of impacts across climate and building use," Renewable and Sustainable Energy Reviews, vol. 82, n. 3, pp. 2300-2318, 2018. DOI: https://doi.org/10.1016/j.rser.2017.08.083

[16] K. Ulgen, "Experimental and theoretical investigation of effects of wall's thermophysical properties on time lag and decrement factor," Energy and Buildings, vol. 32, n. ${ }^{\circ}$ 3, pp. 273-278, 2002. DOI: https://doi.org/10.1016/S0378-7788(01)00087-1 
[17] P. Tuohy et al., "Thermal mass, insulation and ventilation in sustainable housing - an investigation across climate and occupancy," presentado en IX International IBPSA Conference, Montreal, 2005.

[18] S. Ferrari, "Building envelope and heat capacity: re-discovering the thermal mass for winter energy saving," presentado en $2^{\text {nd }}$ Palenc Conference and $28^{\text {th }}$ AIVC Conference on Building Low Energy Cooling and Advanced Ventilation Technologies in the $21^{\text {st }}$ Century, Creta, 2007.

[19] L. Zhu et al., "Detailed energy saving performance analyses on thermal mass walls demonstrated in a zero energy house," Energy and Buildings, vol. 41, n. ${ }^{\circ}$ 3, pp. 303-310, 2009. DOI: https://doi.org/10.1016/j.enbuild.2008.10.003

[20] M. Scott, "Beating the Heat," Nasa Earth Observatory, [En línea], Disponible: http:// earthobservatory.nasa.gov/Features/GreenRoof/, 2006.

[21] D. Campbell-Lendrum y C. Corvalán, "Climate change and developing-country cities: implications for environmental health and equity," Journal of Urban Health, vol. 84, n. 1 , pp. 109-115, 2007. DOI: https://doi.org/10.1007/s11524-007-9170-x 